\title{
Deep Abrasion Resistance of Porcelain Stoneware Tiles: An Updated Look
}

\author{
Maziar Montazerian ${ }^{1,2}$, , Mina Eilaghi ${ }^{2}$, Nirvana Mousavi ${ }^{1}$ \\ ${ }^{1}$ R\&D Center, Apadana Ceram Company, No. 8, Goldasht Alley, Molla Sadra St., 15875-6786, Tehran, Iran \\ ${ }^{2}$ Present addresses: Center for Research, Technology, and Education in Vitreous Materials (CeRTEV), \\ Federal University of São Carlos (UFSCar), São Carlos, SP, 13.565-905, Brazil
}

* Corresponding Author: M. Montazerian

Tel: +55-16-98165.9709

E-mail: $\underline{\text { maziar_montaz@yahoo.com, montazerian@alumni.iust.ac.ir }}$

\begin{abstract}
When we were working on improving the resistance to deep abrasion of porcelain stoneware tiles, several tiles which had a very high resistance to abrasion were found in the market. The abraded volume of these tiles, measured via ISO 10545-6 method, was conspicuously less than $75 \mathrm{~mm}^{3}$. These values were well below the standard limit $(<175$ $\mathrm{mm}^{3}$ ) and reported values in the literature $\left(>110 \mathrm{~mm}^{3}\right)$. We determined the chemical, physical and microstructural characteristics of these tiles and other commercially available ones to find the relationship between the abrasion resistance and the technical specifications. We also characterized the samples by Vickers microhardness (VH), X-ray diffraction (XRD), mercury intrusion porosimetry (MIP) and scanning electron microscopy (SEM). It was observed that the high resistance to abrasion could be due to the presence of an adequate glassy phase capable of wetting all the hard crystalline phases in the matrix such as quartz and mullite. Therefore, the resistance to deep abrasion could be improved by increasing the amount of "vitreous" matrix, without altering the content of "hard" phases considerably well-bonded to the matrix.
\end{abstract}

Keywords: Porcelain; Tile; Abrasion Resistance; Glass 


\section{Introduction}

Porcelain stoneware tiles are well-known technical ceramics which are extensively used for wall cladding and outdoor flooring. Their outstanding properties such as frost, thermal and abrasion resistance along with low porosity and aesthetic appearance distinguish them from traditional and porous tiles. They are categorized into the two groups, glazed and unglazed porcelain [1], [2]. The stringent standards are considered for porcelains because they have been designed for use in harsh environments such as high traffic areas and severe weather conditions. Furthermore, the worldwide annual tile production which is considerable $\left(\sim 15\right.$ million $\mathrm{m}^{2}$ in 2016$)$, encourages the industry to satisfy all customer requirements beyond the standard limits and in an innovative way [3], [4].

The porcelain tiles are characterized by their low water absorption $(\leq 0.5 \%)$, a significant module of rupture $(\geq 35 \mathrm{MPa})$ and low abraded volume $\left(<175 \mathrm{~mm}^{3}\right)$. The glazed tiles have higher resistance to staining and household chemicals due to the protective cover made of vitreous and impervious glazes. However, the unglazed ones, which are usually polished, are susceptible to staining and abrasion [5]. Polishing by SiC abrasive followed by high energy grinding and leveling reveals closed porosity on the surface and results in the formation of intricate texture. The characterization and modification of resultant surface are vital to improving mechanical and surface properties, specifically stain and abrasion resistance [6].

Dondi et al. have shown that the pore morphology and surface roughness are primary factors which actively control the stain resistance [7]. Numerous attempts have been made 
to improve resistance to staining by applying polymer coatings on the surface or increasing the vitreous phase through double charging [8], [9]. Additionally, selective crystallization of hard phases like zircon $\left(\mathrm{ZrSiO}_{4}\right)$ and zirconia $\left(\mathrm{ZrO}_{2}\right)$ may improve abrasion resistance [10]. The phase composition plays a fundamental role, e.g., the more significant is the content of mullite $\left(3 \mathrm{Al}_{2} \mathrm{O}_{3} \cdot 2 \mathrm{SiO}_{2}\right)$, zircon, and quartz $\left(\mathrm{SiO}_{2}\right)$, the higher is the resistance to deep abrasion [11]. In particular, mullite and zircon ensure the main contribution to the tribological properties, owing to both their hardness and a toughening mechanism originated by differences of thermal expansion and elastic moduli between glassy and crystalline phases, inducing a compression at grain boundaries. Other crystalline phases, such as quartz and corundum $\left(\mathrm{Al}_{2} \mathrm{O}_{3}\right)$, have a less relevant effect on the wear resistance, for the unfavorable differences of thermal expansion with the vitreous matrix [11]. We could also envisage that the resistance to abrasion depends on surface microstructure and characteristics such as pore size, chemical analysis, mineral phases, the degree of vitrification, etc. that may influence abrasion resistance.

When we were working on improving the resistance to deep abrasion of porcelain tiles in Apadana Ceram Co. in Iran, we found in the market some tiles which had a very high resistance to abrasion. The abraded volume of these tiles, measured via ISO 10545-6 method, was less than $75 \mathrm{~mm}^{3}$. Figure 1 shows that these values are well below the standard limit $\left(<175 \mathrm{~mm}^{3}\right)$ and reported values $\left(>110 \mathrm{~mm}^{3}\right)$ in Dondi et al. [11]. Therefore, we decided to determine the most influential factors affecting the resistance of commercial porcelain tiles. For that reason, we thoroughly characterized ten commercial unglazed porcelain tiles and explored the correlations between technical features and deep 
abrasion resistance. In each step, we also compared our results with M. Dondi's study [11]. Our studies could help us to employ appropriate methods for improving the abrasion resistance.

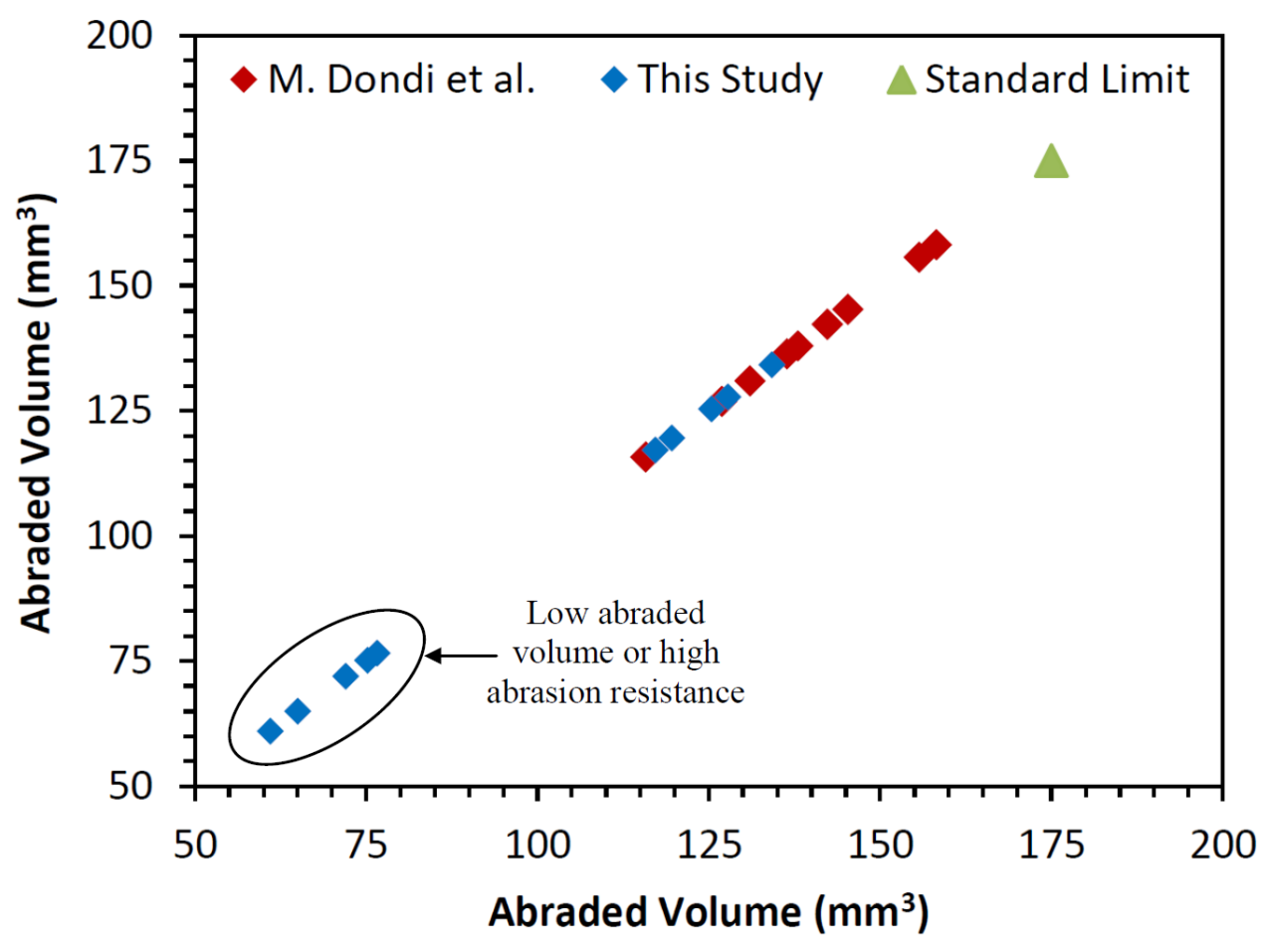

Figure 1. The abraded volume of the samples measured via ISO 10545-6 method.

\section{Experimental procedure}

Ten various kinds of industrially manufactured polished tiles were selected to demonstrate the different range of technology and decoration techniques currently available in the market. Table 1 shows the name and typology of the samples. The tiles were named so that $\mathrm{P} 1$ demonstrates the maximum resistance to abrasion (minimum material removal due to the abrasion). Resistance to abrasion and technical characteristics of all samples 
were determined. We tested at least five samples $\left(10 \times 10 \mathrm{~cm}^{2}\right)$ cut from the original tiles $\left(60 \times 60 \mathrm{~cm}^{2}\right)$ for each kind of analysis.

Deep abrasion resistance (DAR) test was performed by Gabrielli testing machine Model CAP according to ISO 10545-6. The test was conducted using a steel disc (Fe 360 A) with a diameter of $200 \mathrm{~mm}$ and a thickness of $10 \mathrm{~mm}$ rotating over the sample at $75 \mathrm{rpm}$ and 150 revolutions. We used $\mathrm{Al}_{2} \mathrm{O}_{3}$ powder as abrasive media. Hereafter, further analyses were performed on abraded samples. DAR was then defined by the reciprocal of the abraded volume of the material multiplied by 100 as indicated in equation (1).

Deep Abrasion Resistance $(\mathrm{DAR})=\frac{1}{\text { Abraded Volume of Material }} \times 100$

Table 1. Sample names, typology and abrasion resistance.

\begin{tabular}{|c|c|c|c|}
\hline Sample & Typology & $\begin{array}{c}\text { Abraded } \\
\text { Volume } \\
\left(\mathbf{m m}^{\mathbf{3}}\right)\end{array}$ & $\begin{array}{c}\text { Deep Abrasion } \\
\text { Resistance } \\
\left(\mathbf{m m}^{-3} \mathbf{1 0 0}\right)\end{array}$ \\
\hline P1 & Full White Body + Soluble Salts & $61 \pm 9.7$ & $1.64 \pm 0.31$ \\
\hline P2 & Non-chromatic Body & $65 \pm 5.1$ & $1.54 \pm 0.13$ \\
\hline P3 & Double Charged + Micronized Granule & $72 \pm 6.3$ & $1.39 \pm 0.13$ \\
\hline P4 & Full Beige Body + Flaked Granule & $75.2 \pm 5.3$ & $1.33 \pm 0.10$ \\
\hline P5 & Full Dark Body + Double Charged + & $76.6 \pm 8.7$ & $1.31 \pm 0.17$ \\
\hline P6 & Soluble Salts & $117.2 \pm 23.4$ & $0.85 \pm 0.21$ \\
\hline P7 & Non-chromatic Body & $119.6 \pm 17.9$ & $0.84 \pm 0.15$ \\
\hline P8 & Non-chromatic Body + Soluble Salts & $125.4 \pm 15.2$ & $0.80 \pm 0.11$ \\
\hline P9 & Super White Body + Double Charged + & $127.8 \pm 3.9$ & $0.78 \pm 0.02$ \\
\hline P10 & Micronized White Granule & $134.2 \pm 3.9$ & $0.75 \pm 0.02$ \\
\hline
\end{tabular}


We used a Vickers microhardness tester with a diamond pyramid indenter (Buehler, Micromet I) to measure microhardness. The load was $500 \mathrm{~g}$ and the loading time was $20 \mathrm{~s}$. Data of hardness were determined using at least ten indentations on each specimen and near the abraded area.

Bulk density, apparent porosity, and water absorption were determined by water saturation under vacuum and Archimedes' principle (ISO 10545-3). The powder density was measured by pycnometer helping us to calculate closed porosity together with relative density. Pore size distribution estimated by mercury intrusion porosimetry (ThermoFinnigan Pascal 140) on tile fragments with an apparent area of around $3 \mathrm{~cm}^{2}$.

The chemical composition of powdered tiles was determined by wet chemical analysis. The samples were then subjected to X-ray diffraction (XRD) analysis using a powder diffractometer (Philips XPert, Co $\mathrm{K}_{\alpha}$ radiation at $40 \mathrm{kV}$ ). Silicon powder (10 wt\%) was used as the standard for semi-quantitative measurements. J. Martín-Márquez et al. [12] have shown that a straightforward and feasible quantitative XRD analysis is possible when the intensity of a particular diffraction peak for each crystalline phase is compared with a reference material. This technique is a suitable and feasible methodology for people working in the industry to obtain preliminary knowledge on an amount of mineral phases evolved in porcelain stoneware. Therefore, the peak intensity of particular phases such as quartz, mullite, and albite was determined using OriginPro 7.5 SR0 software. The intensity of peaks was then compared with the peak intensity of $\mathrm{Si}$ to minimize any possible 
systematic errors. We did microstructural studies by scanning electron microscope (SEM) working in the secondary electron (SE) mode (SEM, Philips XL30).

\section{Results}

The deep abrasion resistance (DAR) and other technical characteristics of the porcelain tiles are summarized in Table 2. The correlations between DAR and porosity content, bulk density, mean pore size, and microhardness are also plotted in Figures 2(a)(d), respectively. The trending lines related to correlation factors of $\mathrm{R}^{2}>0.9$ are also illustrated. To fit a better trending line, we considered sample P4 and in one case sample P7 (red crosses in Fig. 2d) as outliers, but they follow, in general, the trends. Additionally, the reported values for DAR by M. Dondi et al. [11] are included in the Figures (red points).

Table 2. Abrasion resistance and technical characteristics of the samples (apparent porosity of all samples was below $0.5 \%$ ).

\begin{tabular}{|c|c|c|c|c|c|}
\hline Sample & $\begin{array}{c}\text { Deep Abrasion } \\
\text { Resistance } \\
\left(\mathbf{D A R}, \mathbf{~ m m}^{-\mathbf{3}} \mathbf{\times 1 0 0}\right)\end{array}$ & $\begin{array}{c}\text { Closed } \\
\text { Porosity } \\
(\mathbf{\%})\end{array}$ & $\begin{array}{c}\text { Bulk } \\
\text { Density } \\
\left(\mathbf{g} / \mathbf{c m}^{\mathbf{3}}\right)\end{array}$ & $\begin{array}{c}\text { Mean } \\
\text { Pore Size } \\
(\boldsymbol{\mu m})\end{array}$ & $\begin{array}{c}\text { Hardness } \\
(\mathbf{G P a})\end{array}$ \\
\hline P1 & $\mathbf{1 . 6 4} \pm \mathbf{0 . 1 1}$ & $9.50 \pm 0.14$ & $2.28 \pm 0.01$ & $4.5 \pm 0.2$ & $4.61 \pm 0.67$ \\
\hline P2 & $\mathbf{1 . 5 4} \pm \mathbf{0 . 1 3}$ & $8.30 \pm 0.17$ & $2.31 \pm 0.01$ & $4.6 \pm 1.2$ & $4.68 \pm 0.71$ \\
\hline P3 & $\mathbf{1 . 3 9} \pm \mathbf{0 . 1 3}$ & $5.60 \pm 0.12$ & $2.36 \pm 0.01$ & $3.4 \pm 0.7$ & $5.43 \pm 0.80$ \\
\hline P4 & $\mathbf{1 . 3 3} \pm \mathbf{0 . 1 0}$ & $8.30 \pm 0.10$ & $2.32 \pm 0.01$ & $12.4 \pm 3.1$ & $4.65 \pm 0.89$ \\
\hline P5 & $\mathbf{1 . 3 1} \pm \mathbf{0 . 1 7}$ & $5.60 \pm 0.21$ & $2.37 \pm 0.02$ & $6.3 \pm 1.5$ & $5.59 \pm 0.55$ \\
\hline P6 & $\mathbf{0 . 8 5} \pm \mathbf{0 . 2 1}$ & $8.3 \pm 0.13$ & $2.32 \pm 0.03$ & $14.2 \pm 2.1$ & $6.13 \pm 0.78$ \\
\hline P7 & $\mathbf{0 . 8 4} \pm \mathbf{0 . 1 5}$ & $10.3 \pm 0.41$ & $2.27 \pm 0.02$ & $36.6 \pm 9.6$ & $3.94 \pm 0.91$ \\
\hline P8 & $\mathbf{0 . 8 0} \pm \mathbf{0 . 1 1}$ & $7.5 \pm 0.19$ & $2.34 \pm 0.01$ & $12.1 \pm 2.2$ & $5.39 \pm 0.97$ \\
\hline P9 & $\mathbf{0 . 7 8} \pm \mathbf{0 . 0 2}$ & $6.6 \pm 0.14$ & $2.41 \pm 0.02$ & $68.7 \pm 10.2$ & $5.34 \pm 0.85$ \\
\hline P10 & $\mathbf{0 . 7 5} \pm \mathbf{0 . 0 2}$ & $7.9 \pm 0.17$ & $2.31 \pm 0.01$ & $28.9 \pm 4.5$ & $5.05 \pm 1.08$ \\
\hline
\end{tabular}


The apparent porosity (open porosity) for all samples was less than $0.5 \%$. Interestingly, the abrasion resistances are divided into the two ranges of $1.3-1.7\left(\mathrm{~mm}^{-}\right.$ $\left.{ }^{3} \times 100\right)$ for samples P1-P5 and 0.75-0.85 $\left(\mathrm{mm}^{-3} \times 100\right)$ for P6-P10. The DAR of samples P6P10 is less sensitive to the variation in the technical features of tiles (the slope of the trend lines is close to zero). We were much more interested in understanding the reason for the high DAR of the tiles in the former group. It was our objective to correlate this high DAR to the technical specifications of the tiles. This study could help us to reach high values of DAR (more than 0.85) after knowing the relevant and effective parameters. M. Dondi's group [11] has also reported the same range of characteristics for those tiles which have low resistance to abrasion (Figure 2). They have studied this group extensively [11].

Samples P1-P5 shows the highest resistance to abrasion and their DAR changes more markedly than group P6-P10. The former group drew our attention. When their porosity content increases, the bulk density decreases, as expected (comparing Figs. 2a and 2b). Surprisingly, their DAR increases with increasing the porosity content (Fig. 2a) or decreasing bulk density (Fig. 2b). DAR also drops when the pores become larger (Fig. 2c), or the samples become softer (Fig. 2d). Furthermore, these Figures reveal two main differences between these two series of samples. First, samples with high DAR have a remarkably smaller pore size of less than $10 \mu \mathrm{m}$ (Fig. 2c), second, their DAR decreases with increase in microhardness unlike of another group which shows a slight increasing trend for DAR vs. microhardness (Fig. 2d). 

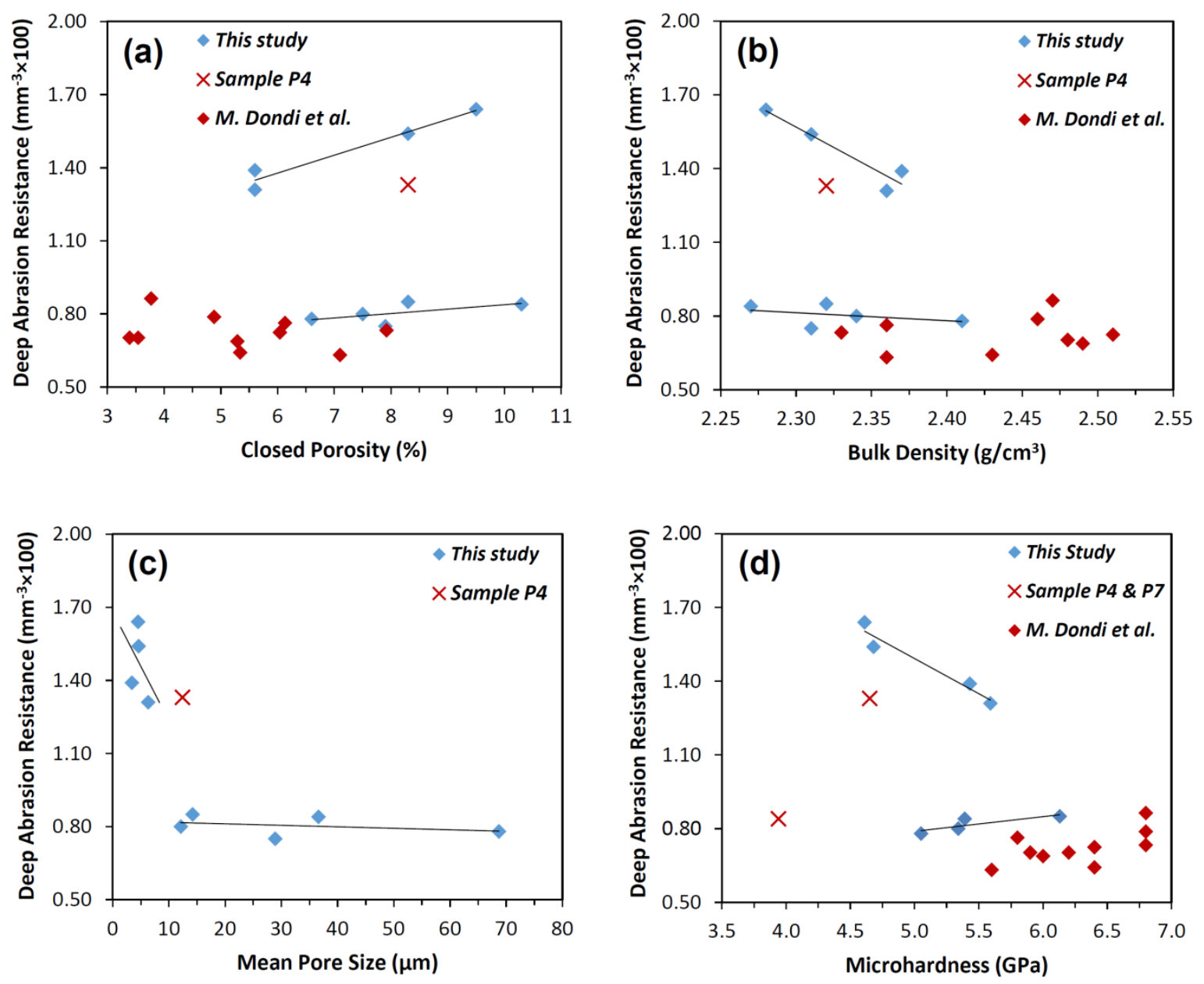

Figure 2. Correlation between abrasion resistance and (a) porosity, (b) bulk density, (c) mean pore size, and (d) hardness. We considered sample P4 and, in one case, P7 as an outlier to fit better trend lines $\left(\mathrm{R}^{2}>0.9\right)$ for the samples studied in this paper.

The chemical composition of the specimens is summarized in Figure 3. The main constituents of the samples, which are $\mathrm{SiO}_{2}, \mathrm{Al}_{2} \mathrm{O}_{3}, \mathrm{Na}_{2} \mathrm{O}$, and $\mathrm{K}_{2} \mathrm{O}$ could be supplied from quartz, kaolinite clays (kaolin or ball clays) and fluxes like Na/K-feldspars. Samples P5 and P9 had a few amounts of zircon as a whitening agent. 


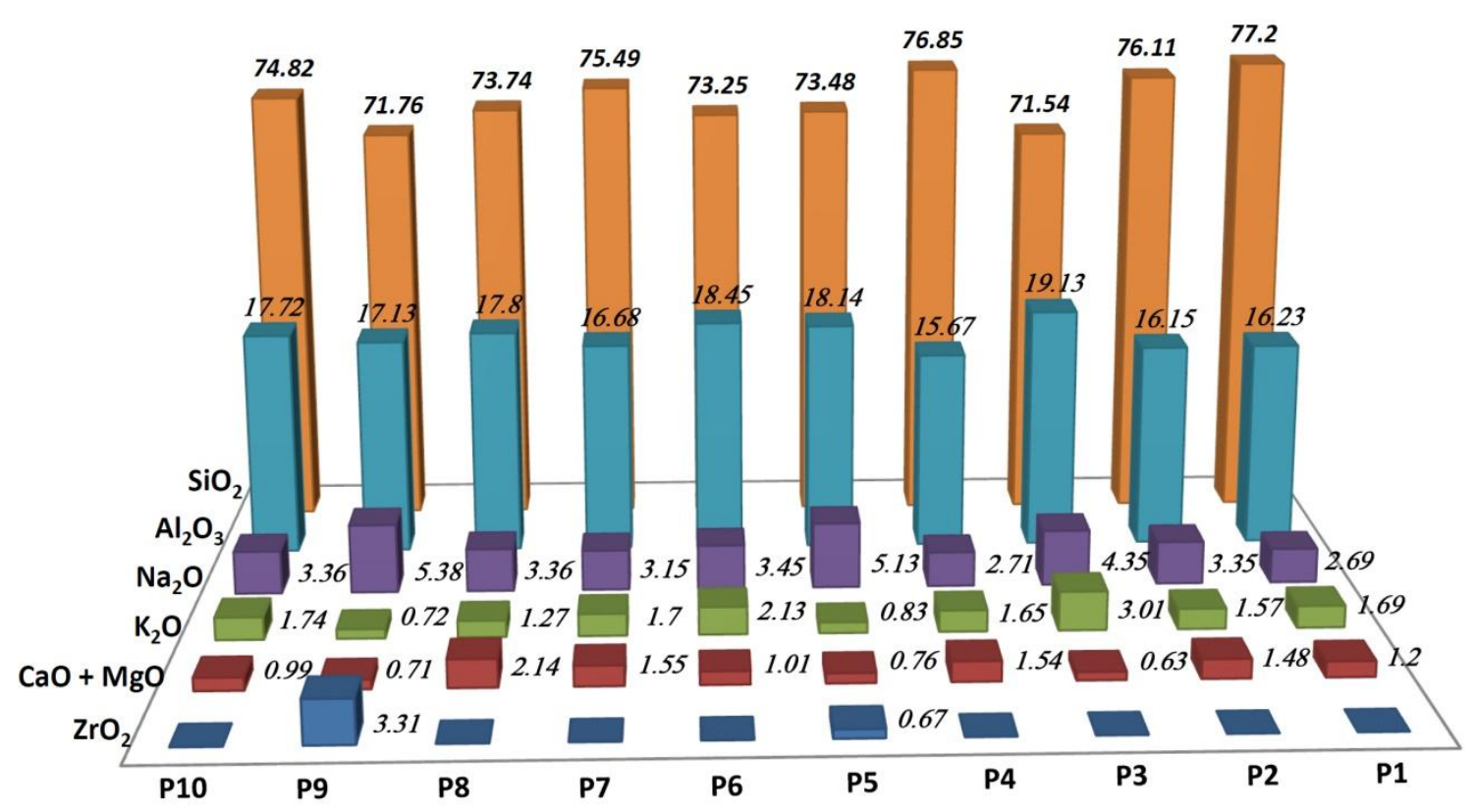

Figure 3. Chemical analysis (wt $\%)$ of the samples $\left(\mathrm{Fe}_{2} \mathrm{O}_{3}+\mathrm{TiO}_{2}<1.4 \%\right.$ for all samples).

XRD graphs for samples P1-P5 and P6-P10 are shown in Figures 4 (a) and (b), respectively. The identified phases are also summarized in Table 3. As can be observed, samples P1-P5 mainly contain quartz, mullite, amorphous phase and a minor amount of Nafeldspar. However, the Na-feldspar becomes a dominant phase in samples P6-P10. Also, sample P9 as a super white tile contains zircon. To semi-quantify the amount of phases in the samples, the main peak intensity of each phase was compared with the Si peak intensity $\left(\mathrm{I}_{\mathrm{Si}(111)}\right)$. We considered the intensity of the baseline as a criterion for the amount of the amorphous phase. The results are summarized in Table 3. We should emphasize here that this semi-quantifying XRD method proposed by Martín-Márquez et al. [12] is easy to perform in the industry, is cheaper and faster than more complicated techniques, e.g., Rietveld refinement. However, this method is only useful when we want to compare two 
samples in relation to the amount of phases they contain. Finally, the correlations between abrasion resistance and the amount of identified phases are drawn in Figures 5 (a)-(d), based on the values of Table 3 .
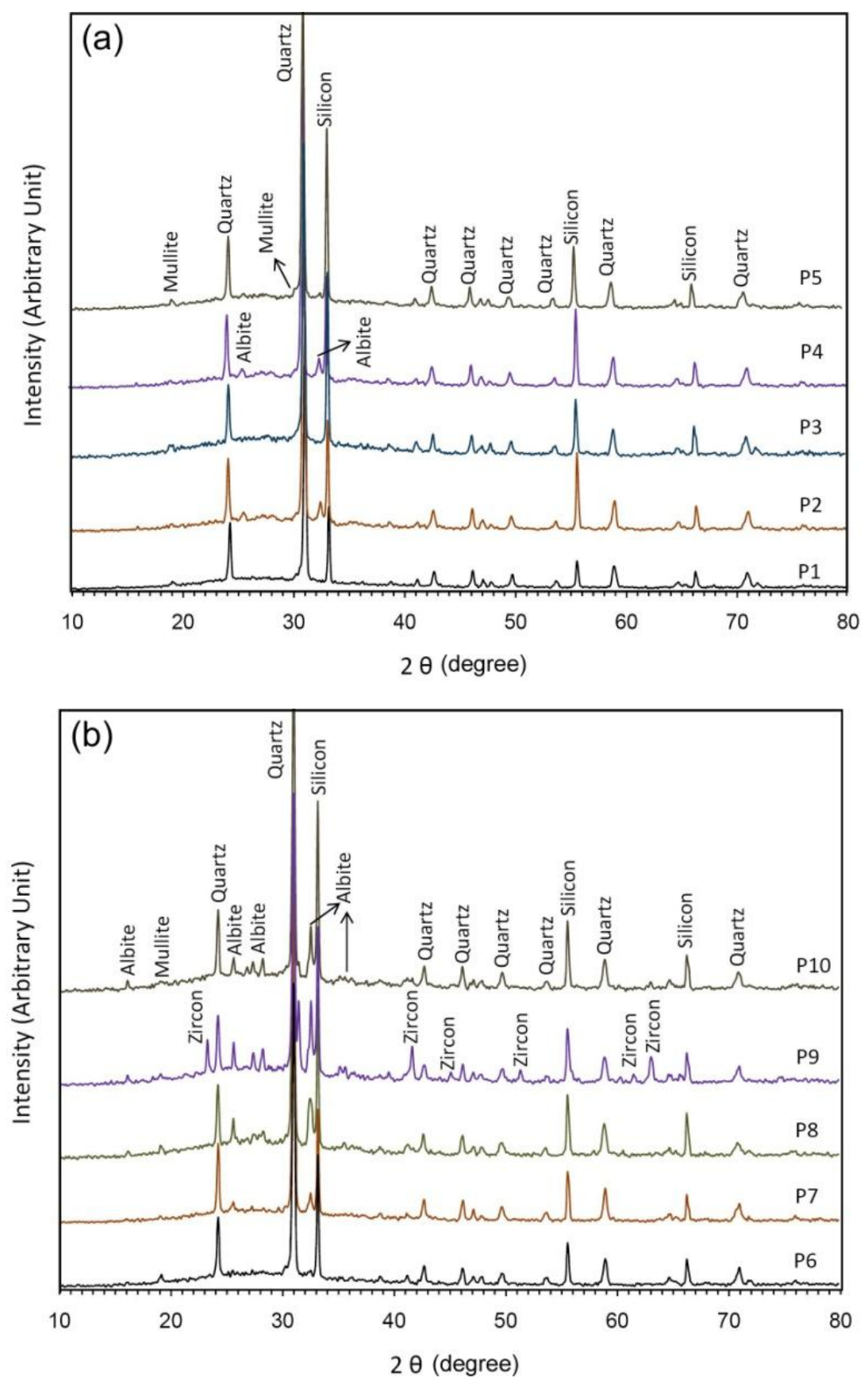

Figure 4. XRD analysis of (a) Samples P1-P5 and (b) samples P6-P10. 
Table 3. Identified phases by XRD analysis.

\begin{tabular}{|c|c|c|c|c|c|}
\hline Sample & Phases & $\frac{\mathbf{I}_{\text {Quartz(101) }}}{\mathbf{I}_{\mathbf{S i}(\mathbf{1 1 1})}}$ & $\frac{\mathbf{I}_{\text {Mullite(110) }}}{\mathbf{I}_{\mathbf{S i}(\mathbf{1 1 1})}}$ & $\frac{\mathbf{I}_{\text {Albite(002) }}}{\mathbf{I}_{\mathbf{S i} \text { (111) }}}$ & $\frac{\mathbf{I}_{\text {BaseLine }}}{\mathbf{I}_{\mathbf{S i}(\mathbf{1 1 1})}}$ \\
\hline P1 & Quartz + Mullite & 2.07 & 0.2 & 0 & 0.46 \\
\hline P2 & Quartz + Mullite* + Albite & 1.85 & 0.1 & 0.12 & 0.41 \\
\hline P3 & Quartz + Mullite & 1.38 & 0.14 & 0 & 0.34 \\
\hline P4 & Quartz + Mullite* + Albite & 1.71 & 0.1 & 0.24 & 0.44 \\
\hline P5 & Quartz + Mullite + Albite* & 1.31 & 0.13 & 0.1 & 0.34 \\
\hline P6 & Quartz + Mullite + Albite & 1.6 & 0.18 & 0.15 & 0.43 \\
\hline P7 & Quartz + Mullite* + Albite & 1.75 & 0.08 & 0.34 & 0.4 \\
\hline P8 & Quartz + Mullite + Albite & 1.44 & 0.14 & 0.46 & 0.35 \\
\hline P9 & Quartz + Mullite + Albite + Zircon & 1.41 & 0.12 & 0.62 & 0.34 \\
\hline P10 & Quartz + Mullite* + Albite & 1.32 & 0.1 & 0.47 & 0.32 \\
\hline
\end{tabular}

\section{* Minor Phases}

It is evident in Figures 5 (a) and (b) that by increasing the quartz and mullite contents, the DAR increases. Additionally, it seems that by increasing the amount of unmelted albite (Fig. 5c) or reducing the content of the glass phase in the fired tiles (Fig. 5d), abrasion resistance significantly decreases. 

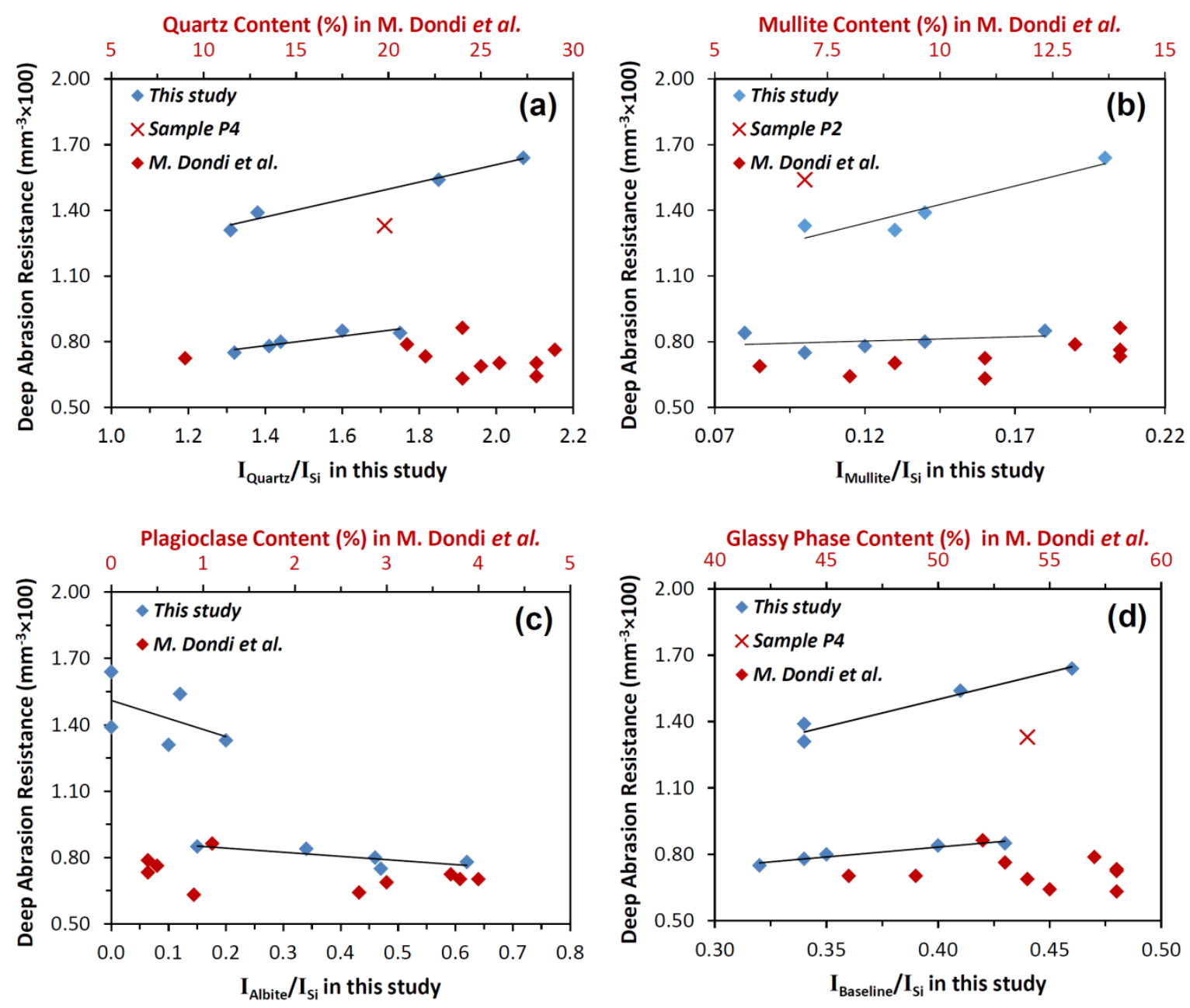

Figure 5. Correlation between abrasion resistance and the amount of identified phases. Abrasion resistance versus the content of (a) quartz, (b) mullite, (C) albite and (d) amorphous phases. Sample P4 was considered an outlier to fit better trend lines in this study $\left(\mathrm{R}^{2}>0.9\right)$.

SEM micrograph of sample P1, P5, and P8 are shown in Figures 6 (a)-(c), respectively. The well-vitrified surface of sample P1 and P5 could be observed in Figures 6 (a) and (b). However, sample P8 has a complex microstructure containing various sizes of 
micropores and also quartz particles, which are not well bonded in the matrix. Figure 6 (d) shows quartz particles surrounded by pores and glass phase.
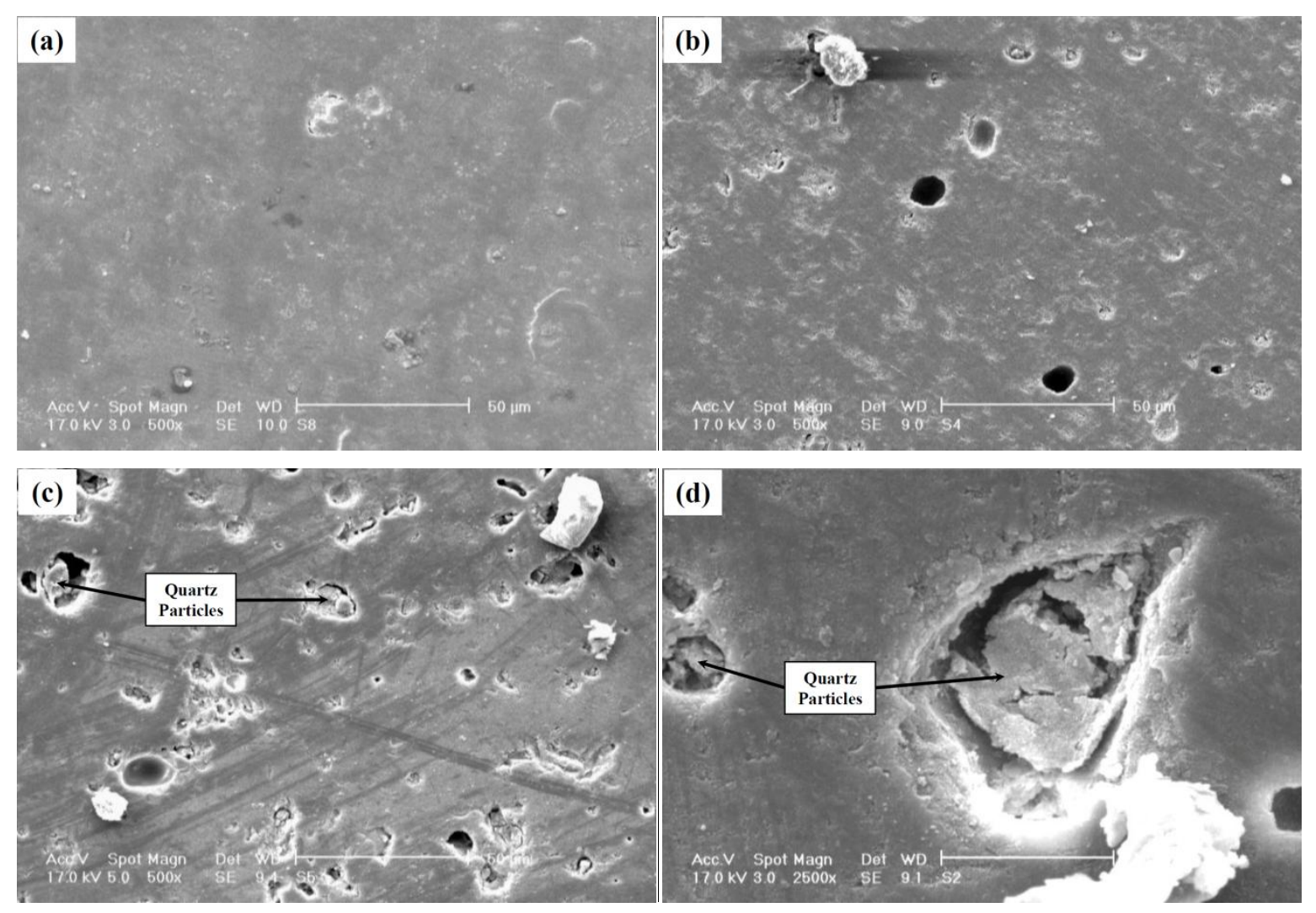

Figure 6. SEM micrograph of samples (a) P1, (b) P5 and (C \& d) P8 (Mag. $\times 500$ for a, b and c \& Mag. $\times 2500$ for d).

\section{Discussion}

Surprisingly, we observed that DAR increases with decreasing bulk density (Fig. 2b). DAR also increases when the pores become smaller (Fig. 2c) or the samples become softer (Fig. 2d). Furthermore, Figures 5 (a) and (b) show that quartz and mullite could increase the DAR. However, the unmelted albite (Fig. 5c) and low amount of glassy phase (Fig. 5d) are detrimental for DAR. We could predict that these trends are the consequence of the 
role of glassy/vitreous phase presented in the samples. Then, we tried to prove this hypothesis through looking carefully at the phase evolution process during firing of porcelain tiles, from the early stage of sintering (lower temperatures) to the complete formation of new phases (higher temperatures).

J. Martín-Márquez et al. [12] selected a mixture of 50\% kaolinitic clay, 40\% feldspar, and $10 \%$ quartz as a representative composition of commercial porcelain stoneware tiles produced via a conventional fast-firing process. Then, they studied the phase evolution during fast firing. After the qualitative and quantitative characterization of mineral and amorphous phases present in the body after firing in the $400-1400{ }^{\circ} \mathrm{C}$ interval, they concluded that:

(1) The fluxing constituents start to decompose at low temperatures (400-800 ${ }^{\circ} \mathrm{C}$ interval) and are completely dissolved above $1200{ }^{\circ} \mathrm{C}$ leading to the virtually zero $(<0.5 \%)$ open porosity and a low level of closed porosity $(<10 \%)$.

(2) Quartz particles, which promote thermal and dimensional stability thanks to its high melting point, start to dissolve in the formed glassy phase around $1230{ }^{\circ} \mathrm{C}$.

(3) As a result of the above remarks, the amorphous phase continuously increases in the complete range of firing temperature.

(4) Mullite is first formed in the porcelain stoneware sample fired above $1100{ }^{\circ} \mathrm{C}$ and increases up to $1230{ }^{\circ} \mathrm{C}$. At higher temperatures, the percentage of mullite remains constant. 
(5) Finally, the mineralogical composition of porcelain stoneware bodies fired in the 1230$1240{ }^{\circ} \mathrm{C}$ range was found to be approximately $61 \%$ glassy phase, $24 \%$ quartz, and $14 \%$ mullite.

Therefore, porcelain tile is the polycrystalline materials consisted of a glassy matrix which embeds quartz particles and mullite phase. Some may call this material a kind of composite. In this somewhat complicated microstructure, more vitreous phase (Fig. 5d), which means less unmelted fluxes such as albite (Fig. 5c), could decrease bulk density (Fig. 2b), mean pore size (Fig. 2c) and hardness (Fig. 2d), and finally resulted in higher DAR. In general, the glassy phase has a lower density, viscous flowability, and hardness than rigid inclusions like quartz and mullite [1].

It is known that stress and formation of micro-pores near the quartz particles strongly influence the mechanical and surface properties. This results from thermal expansion coefficient mismatch between quartz particles and glassy matrix. Additionally, viscosity and the amount of amorphous phase at firing temperature play a pivotal role to enhance densification and reduce defects [7], [8]. The high amount of low-viscous amorphous phase helps to promote densification and also relieve residual stress during the firing cycle, resulting in the improved mechanical properties such as toughness and strength [8], [13], [14]. It seems that, in this study, a higher amount of glassy phase in samples P1-P5 could adequately wet all the hard crystalline phases like quartz and mullite, as we could observe well-vitrified surfaces of P1 and P5 in Figures 6a and 6b. However, the quartz particles have shown lacks of cohesion with the matrix in the sample, e.g., P8 (Figs. 6c and 6d) are easily removed during abrasion. Therefore, the tailor-made vitrified surface could be highly 
suggested for improving resistance to abrasion. This could be achieved by employing a higher firing temperature or using more reactive fluxes in the body composition. It seems that in this condition a vitreous phase which has high resistance to cracking is preferred. Rosales-Sosa et al. [15] reported that the composition of $60 \mathrm{Al}_{2} \mathrm{O}_{3} \cdot 40 \mathrm{SiO}_{2}$ glass, which is identical to that of mullite, has excellent high cracking resistance with high elastic moduli and Vickers hardness. Their results indicate that there exist aluminosilicate glassy compositions (e.g., vitreous phases formed in porcelain tiles) that can produce damagetolerant glasses [15].

In recent years, companies prefer to use fluxing agents such as frits, nepheline syenite, Li-Feldspar, etc. to enhance glass phase formation and finally improve mechanical properties and resistance to staining [16], [17]. In this study, sample P1-P5 had wellvitrified surface possibly resulted from high firing temperature or using fluxes in the second charge. On the other hands, samples P6-P10 had a higher content of residual Na-Feldspar (Fig. 5c), which was not melted during firing leading to the low amount of glass phase. Therefore, they were more sensitive to material removal than sample P1-P5.

\section{Conclusions}

Our analysis of deep abrasion resistance of commercial porcelain tiles revealed that high resistance to abrasion was represented by samples with a high content of the glassy phase and a low amount of non-melted Na-feldspar. Surface textures having a high content of micro-pores and well not embedded quartz particles were much sensitive to abrasion. Therefore, the tailor-made vitrified surface could be highly suggested for improving 
resistance to abrasion. This objective could be achieved by employing a higher firing temperature or using more reactive fluxes in the body formulation or second charges. It seems that, in this condition, the presence of hard phases in a resistant-to-cracking glassy matrix leads to high abrasion resistance.

\section{Acknowledgment}

The authors are grateful to Parvin Alizadeh (Tarbiat Modares University), Hajar Mafi and Maryam Shahriary (Apadana Ceram Company) for their help and support.

\section{References}

[1] G. Biffi, Porcelain Stoneware, Gruppo Editoriale Faenza (Ed.), 1997.

[2] W.M. Carty, U. Senapati, Porcelain - raw materials, processing, phase evolution and mechanical behavior, Journal of the American Ceramic Society 81 (1998) 3-20.

[3] E. Sánchez, J. García-Ten, V. Sanz, A. Moreno, Porcelain tile: Almost 30 years of steady scientific-technological evolution, Ceramics International 36 (2010) 831-845.

[4] L. Baraldi, World production and consumption of ceramic tiles, Ceramic World Review 118 (2016) 68-80.

[5] A.M. Berto, Ceramic tiles: Above and beyond traditional applications, Journal of the European Ceramic Society 27 (2007) 1607-1613.

[6] L. Esposito, A. Tucci, D. Naldi, The reliability of polished porcelain stoneware tiles, Journal of the European Ceramic Society 25 (2005) 1487-1498.

[7] M. Dondi, G. Ercolani, G. Guarini, C. Melandri, M. Raimondo, E. Rocha e Almendra, P.M.T. Cavalcante, The role of surface microstructure on the resistance to stains of porcelain stoneware tiles, Journal of the European Ceramic Society 25 (2005) 357-365 
[8] E. Sánchez, M.J. Ibáñez, J. García-Ten, M.F. Quereda, Y.M. Xu, I.M. Hutchings, Porcelain tile microstructure: Implications for polished tile properties, Journal of the European Ceramic Society 26 (2006) 2533-2540.

[9] L. Carbajal, F. Rubio-Marcos, M.A. Bengochea, J.F. Fernandez, Properties related phase evolution in porcelain ceramics, Journal of the European Ceramic Society 27 (2007) 4065-4069.

[10] F. Bondioli, T. Manfredini, M. Giorgi, G. Vignali, Functionalization of ceramic tile surface by soluble salts addition: Part I, Journal of the European Ceramic Society 30 (2010) 11-16.

[11] M. Dondi, G. Guarini, C. Melandri, M. Raimondo, P.M.T. Cavalcante, C. Zanelli, Resistance to deep abrasion of porcelain stoneware tiles: Key factors, Industrial Ceramics 25(2) (2005) 71-78.

[12] J. Martín-Márquez, A.G. De la Torre, M.A.G. Aranda, J.M. Rincón, M. Romero, Evolution with temperature of crystalline and amorphous phases in porcelain stoneware, Journal of the American Ceramic Society 92 (2009) 229-234.

[13] M. Eilaghi, M. Montazerian, B.E. Yekta, Effect of partial substitution of $\mathbf{K}_{2} \mathbf{O}$ for $\mathrm{Na}_{2} \mathrm{O}$ on sintering, crystallization and mechanical properties of $\mathrm{SiO}_{2}-\mathrm{CaO}-\mathrm{K}_{2} \mathrm{O}$ $\mathrm{Na}_{2} \mathbf{O}-\mathrm{CaF}_{2}$ glass-ceramics, Transactions of the Indian Ceramic Society 75(1) (2016) 1 6.

[14] M. Yousefi, P. Alizadeh, B.E. Yekta, F. Molaie, N. Ghafoorian, M. Montazerian, Synthesis and characterization of diopside glass-ceramic matrix composite reinforced with aluminum titanate, Ceramics International 35(4) (2009) 1447-1452.

[15] G.A. Rosales-Sosa, A. Masuno, Y. Higo, H. Inoue, Crack-resistant $\mathbf{A l}_{2} \mathbf{O}_{3}-\mathbf{S i O}_{2}$ glasses, Scientific Reports 6 (2016) art. no. 23620.

[16] C. Zanelli, G. Baldi, M. Dondi, G. Ercolani, G. Guarini, M. Raimondo, Glassceramic frits for porcelain stoneware bodies: Effects on sintering, phase composition and technological properties, Ceramics International 34 (2008) 455-465.

[17] L. Esposito, A. Salem, A. Tucci, A. Gualtieri, S.H. Jazayeri, The use of nephelinesyenite in a body mix for porcelain stoneware tiles, Ceramics International 31 (2005) 233-240. 